\title{
Standardization of New Airborne Multipath Models
}

\author{
Michael Felux, Mihaela-Simona Circiu, Stefano Caizzone, Markus Rippl, German Aerospace Center (DLR) \\ Matteo Sgammini, European Commission \\ Pièrre Durel, GSA
}

\begin{abstract}
Within the frame of the DUFMAN project funded by the European Commission new multipath models for the new signals are developed in order to be able to exploit the potential benefits for aviation users. Previous papers on the project were addressing the methodology, described the results of the studies and the influence of the antenna. This paper explains the standardization activities and discusses choices that were made in setting up the data collection campaign and the subsequent steps to standardized models. Regarding standardization, the International Civil Aviation Organization (ICAO) is producing Standards and Recommended Practices (SARPS) for DFMC SBAS which will make use of the DFMC multipath models. Further requirements on the hardware exist e.g. in form of Minimum Operational Performance Standards (MOPS) that specify performance of certain components, such as the airborne antenna. A variety of antennas differing significantly in performance is available on the market. Furthermore, the airborne receiver hardware may use different correlator spacing and receiver bandwidth settings which may also have an impact on the results. In the effort to characterize the multipath errors, hardware and processing choices had to be made taking into account all those requirements and the impact on the final models. The paper discusses the interdependency between different standards and explains the choices that were made in the project, as well as results in terms of standardization.
\end{abstract}

\section{INTRODUCTION}

In aeronautical navigation the use of Global Navigation Satellite Systems (GNSS) is becoming ever more important. GNSS are one of the cornerstones of the performance based navigation (PBN) concept. They are currently used for navigation en-route, as well as during arrival procedures and for lateral approach guidance. Together with satellite-based or ground-based augmentation systems (SBAS, GBAS) satellite navigation can already today provide precision approach guidance down to the CAT-I minima (i.e. down to $200 \mathrm{ft}$ above ground). For GBAS, standards for the GPS-based single frequency service type supporting also CAT-III operations are in place [1], allowing the introduction of GNSS-based precision approach guidance in all-weather operations once technical development of ground stations and avionics hardware is completed. In order to ensure sufficient availability of these services and enable new services, such as Advanced Receiver Autonomous Integrity Monitoring (ARAIM), algorithms and systems using multiple GNSS constellations and dual-frequency methods are under development. It is expected that these services can support operations without significant limitations, including in regions with active ionospheric conditions.

On the side of GNSS, all GPS satellites since the Block IIF generation with currently 12 operational satellites provide signals in the L5 band (in addition to the most commonly used signals in the L1 band), a second frequency band usable for aeronautical applications. The Galileo constellation has currently 22 operational satellites in orbit that all provide signals on the E1 and E5a frequency bands. Other constellations, such as Glonass and BeiDou are also launching further satellites so that a large number of navigation satellites are available to users. The use of dual-frequency and multi constellation techniques will mitigate the impact of most ionosphere-related disturbances, significantly increasing service availability.

All GNSS-based navigation methods have in common that they need appropriate integrity concepts safely bounding any residual errors that may prevail in the position solution. With the ionospheric errors largely addressed by dual-frequency and multiconstellation methods, the residual noise and multipath becomes the most significant contributor to the residual errors. In order to bound these errors, standardized error models are used. The existing multipath model was developed based on extensive data analysis, however, using only the legacy GPS signal in the L1 band. Galileo is using a different modulation for the E1 signals which is less susceptible to long-range multipath. The GPS and Galileo signals in the L5/E5a band are using a 10-times higher 
chipping rate than the L1/E1 signal. Therefore, also for these signals, the multipath envelope is significantly smaller, potentially allowing to have smaller error models for these signals. When using dual-frequency methods to remove the ionospheric delay, the receiver tracking noise and multipath error from the signals on both frequencies are combined. For all these cases, the existing standardized GPS L1-based model is not well suited for error modelling and integrity assurance. Therefore, new models bounding the multipath on the Galileo E1, Galileo E5a and GPS L5 signals are developed and brought to standardization. This paper describes the modelling process, the design choices made within the model development and discusses standardization bodies and the ongoing activities.

\section{EXISTING AIRBORNE MULTIPATH MODEL AND STANDARDS}

The ICAO Standards And Recommended Practices (SARPs) [2] assume GPS L1 C/A code measurements to be used for navigation (they also provide models for Glonass, however they are beyond the scope of this work). They describe the airborne error contribution after code-carrier smoothing with a 100s smoothing filter time constant in Section 3.6.5.5.1.1.2 of the SARPs by a zero-mean standard deviation defined as

$$
\sigma_{\text {air }}=\sqrt{\left(\sigma_{\text {receiver }}^{2}(\theta)+\sigma_{\text {multipath }}^{2}(\theta)\right)}
$$

The model depends on the elevation angle $\theta$ of the satellite and consists of a "receiver contribution and a standard allowance for airframe multipath" [2]. The performance requirement for the airborne receiver accuracy is specified to be

$$
R M S_{p r_{-} \text {air }}(\theta) \leq a_{0}+a_{1} \cdot e^{-\left(\theta / \theta_{0}\right)}
$$

Two different performance classes for the airborne accuracy specify different values for the parameters $a_{0}, a_{1}$ and $\theta_{0}$. The aircraft accuracy designator (AAD) " $\mathrm{B}$ " specifies the more stringent performance with $a_{0}=0.11 \mathrm{~m}, a_{1}=0.13 \mathrm{~m}$ and $\theta_{0}=4^{\circ}$. The standard allowance for the airframe multipath is given as

$$
\sigma_{\text {multipath }}(\theta)=0.13+0.53^{-\frac{\theta}{10^{\circ}}}
$$

This multipath model is considered to be representative for all airframes.

In the Minimum Operational Performance Standards (MOPS) for different applications similar performance requirements exist. The MOPS for GPS/SBAS Airborne Equipment (DO-229E) [3] in Appendix J.4.2 and for GBAS Airborne Equipment (DO-253D) [1] in Section 2.3.12.1 specify the same multipath model and detail the noise model further into performance requirements at maximum and minimum signal level. The airborne error model also foresees a budget for the ionospheric divergence rate. In DO253D it is specified that the combined noise and divergence error correspond to the $\sigma_{\text {receiver }}$-term in the ICAO SARPS from Equation (1). These parameters are, however, beyond the scope of this study on airborne multipath.

The newly developed MOPS for Galileo/GPS SBAS Airborne Equipment (ED-259) [4] in Section 3.1.1.6.5.1.2 specify the same multipath model as above. However, this standard explicitly states that the multipath model from Equation (3) includes the antenna group delay variation (AGDV) in addition to the airborne multipath. It is furthermore assumed that the same multipath model applies for the GPS L1, L5 and Galileo E1 and E5a and that the error model for the ionosphere-free combination of the signals is therefore

$$
\sigma_{M P \& A G D, \text { Ifree }}=2.59 \cdot \sigma_{M P \& A G D V, G P S L 1}
$$

implying that the multipath and AGDV are identically distributed, independent and uncorrelated. It is furthermore stated, that "the multipath and AGDV error for unsmoothed measurements is described by a normal distribution with zero mean and a standard deviation ten times greater than for 100-second smoothed measurements. The multipath and AGDV standard deviation models are valid down to 2 degrees." [4]. A note states that the validation of these assumptions is underway.

The existing GPS L1 multipath model was derived based on a large-scale data collection and evaluation campaign with the details described e.g. in [5] and [6]. For the development of new models for the new signals and the ionosphere-free combination of the signals, the methodology was critically reviewed and slightly adapted. The details are discussed in the next section. 


\section{METHODOLOGY FOR MODEL DEVELOPMENT}

The methodology for the development of the new dual frequency GPS and Galileo airborne multipath models was presented and discussed in [7] and [8] and presented to the ICAO NSP, RTCA SC-159 and Eurocae WG-62. The methodology consists of two main branches: a simulation-based approach and a measurement-based approach with a matching of both methods.

The simulation-based approach aims at developing a detailed understanding of all contributing factors to the airborne multipath (discussed in the next section) and developing simulation capabilities to reliably predict performance for a specific installation. This is especially important when assessing if a new hardware/airframe combination that was not part of the data collection in this project, also complies with the developed models. The simulation-based approach takes into account the performance of a specific antenna and its installed performance on a given aircraft including signal reflections on the airframe. Details about the simulation based approach are given in [9].

The measurement-based approach aimed at collecting airborne GNSS measurements with representative hardware. Within the project different single-aisle and wide-body aircraft were used for data collection. The GNSS antenna was in its primary location on the aircraft. The choice of the antenna to be flown is discussed in the section about design choices later on in this paper. The bulk of the flight data was recorded with an RF recorder for later replay to avionics receivers in the lab with the possibility to change receiver parameters. Some data was also recorded with a dual-frequency GPS/Galileo prototype multi-mode receiver (MMR) in the test flights. The outputs of the MMRs (from the flights and the lab replays) were then evaluated. In a first step the code-minus-carrier measurements after mean removal to account for the carrier phase ambiguities were used as estimates for the multipath. Next, the AGDV was calibrated in order to obtain a multipath model excluding antenna contributions. This was possible for the test setup as the antenna was measured in a compact test range before installation on the aircraft. Furthermore, attitude information of the aircraft throughout its flight was used to remove the antenna group delay errors. Then data was sorted into elevation bins. For each elevation bin the data was then modeled by a zero-mean Gaussian overbound to account for potential nonGaussian distribution of the data in the bins. In a final step, an inflation factor to account for the uncertainty in the estimation of the standard deviation due to a limited number of samples was proposed. As the number of available samples is small that step is omitted for the time being. The methodology and all the above shortly summarized steps were described in detail in [8]. Finally, a model describing the standard deviation of the multipath as a function of elevation is derived.

\section{CONTRIBUTING ERROR SOURCES AND DESIGN CHOICES IN MODEL DEVELOPMENT}

One of the key tasks in the project was to understand the contribution to the overall airborne errors of different error sources. From previous studies the main contributing factors that were identified are the airframe, the performance of the antenna used, the receiver with its specific parameters and tracking properties and the subsequent smoothing of the measurements [10],[11].

\footnotetext{
Airframe:

Background: Previous studies were conducted with different aircraft of DLR's research fleet, mainly the Airbus A320 and the DO228, a twin engine propeller aircraft. The results indicated that the amount of multipath could be different as the distance between the antenna and reflecting parts of the airframe changes. However, the installations were experimental and in locations more prone to multipath than the primary locations of the GNSS antennas.
}

Standards: Currently, there is no restriction on the use of the model to certain aircraft types or categories. The GBAS and SBAS MOPS in their latest versions contain a note clarifying that the "multipath error model was developed and validated using flight test data collected on a variety of large, fixed-wing aircraft." [1],[3].

Design choices in the project: In the project it was decided to gather data from as many different airframes as possible, ideally covering the whole range of aircraft likely to use the models. The study now includes data from Airbus A320 and A321 as singleaisle aircraft, A330 as wide-body aircraft with conventional materials and the A350 as wide-body aircraft mostly made of composite materials. In order to obtain representative data, data was collected with the antenna installed in its primary location.

For further comparison and analysis, also data of different aircraft and aircraft categories were analyzed (a Falcon FA-20 business jet and a DO-228 twin-engine propeller aircraft). However, the data is not used in the modelling process as it was collected with 
experimental equipment and experimental antennas that are not completely representative of results in real-world operations. Figure 1 shows the obtained results for four different aircraft types for which data with the same antenna type were available. The aircraft were the A321, A330 and A350 and the Falcon FA 20. For all signals, the three big aircraft are quite similar in performance, while the FA20 shows significantly higher levels of multipath. This might be an effect from the location where the antenna was mounted (experimental antenna on the FA 20) but many of the reflections are likely coming for the aircraft's tail that is closer to the antenna than on the larger aircraft and likely causing significant reflections to its location above the fuselage. Due to the simulation capabilities it is, however, now possible to determine the expected level of noise and multipath for a specific antenna on a given airframe in order to verify the applicability of the model to one installation.

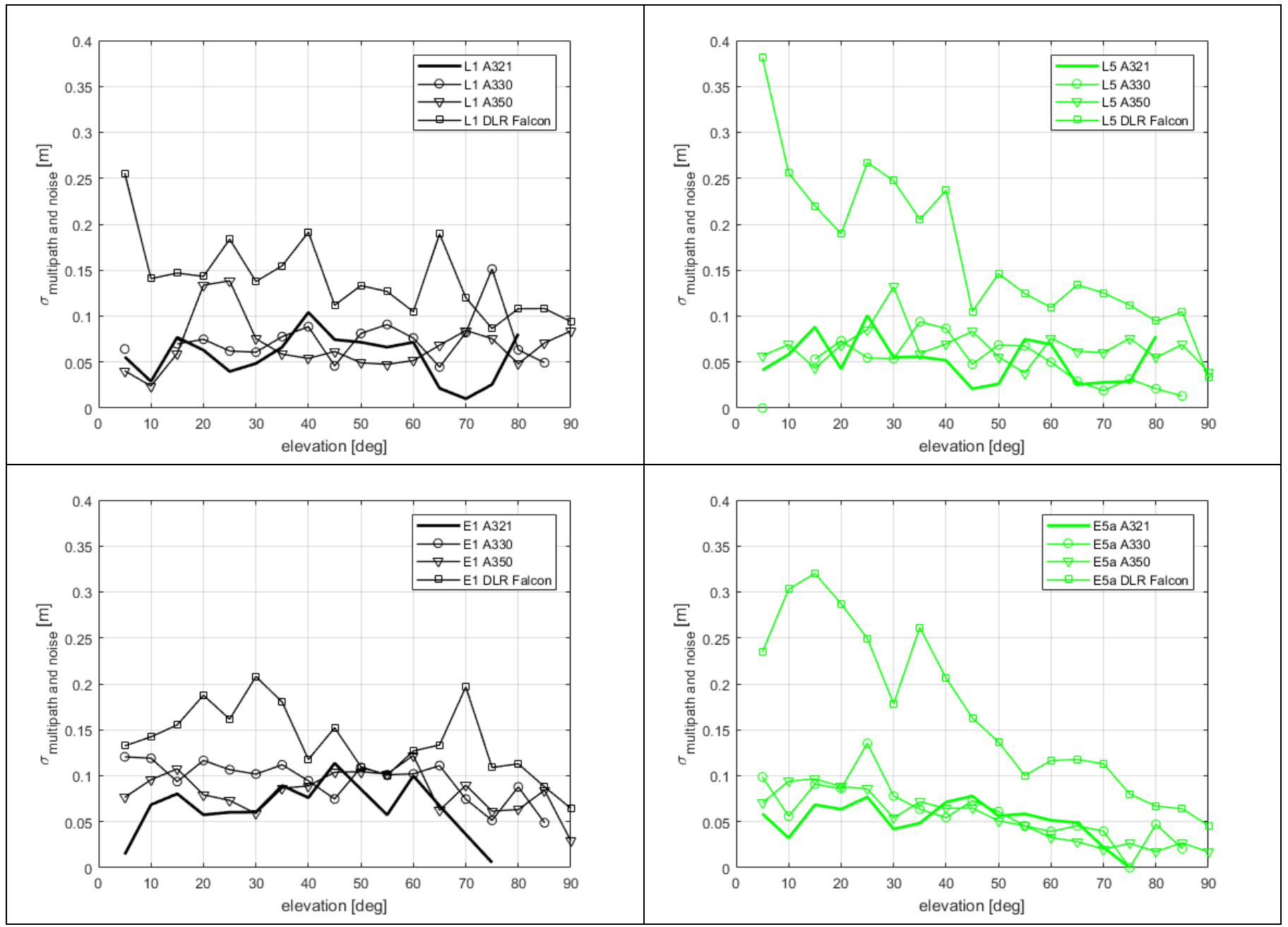

Figure 1 Airborne multipath curves for different airframes. All plots show the multipath as seen on A321, A330 and A350. The upper plots show the results for GPS (L1 left, L5 right), the lower plots for Galileo (E1 left, E5a right)

\section{Antennas:}

Background: There are two main characteristics of antennas that have a significant impact on the multipath performance: the multipath rejection capability and the antenna group delay variation (AGDV). The multipath rejection capability is given by how well the antenna is able to discriminate between right and left hand polarized signals. The AGDV is describing how much variation across all arrival angles there is in the delay the antenna adds to the incoming signals. The group delay adds a bias to the measurements. If the bias was the same for all measurements it would project into the user clock estimate and not affect the 
position solution. However, a variation of the bias over the antenna pattern leads to different range errors as a function of the arrival angle of the signal.

Standards: The requirements for single frequency antennas are defined by DO-301[12] and for dual-frequency antennas in DO-373 [13], which is the relevant standard in the context of this study. The multipath rejection capability is specified by the axial ratio to a maximum of $3 \mathrm{~dB}$ now extending from boresight down to $40^{\circ}$ of elevation (for DO-301 it was only specified at boresight). The Group Delay for each frequency band is specified to be smaller than $1.5 \mathrm{~ns}$ at $5^{\circ}$ elevation, linearly decreasing to $0.65 \mathrm{~ns}$ for elevations at or above $45^{\circ}$.

Regarding the budget for the antenna group delay variation, according to ED-259, it is considered as part of the multipath model.

Design choices within the project: For the choice of the antenna type some trade-offs had to be made. In general the goal was to fly a minimum compliant antenna to the dual frequency antenna MOPS (DO-373). The main antenna parameters of interest in this respect were the AGDV over the whole antenna pattern and the axial ratio as an indicator of the antenna's multipath suppression capability. Antennas are typically either performing very well for all frequencies (high quality and expensive antennas, not desired within the project to avoid overly optimistic performance results) or optimized for one frequency band. From the products on the market one antenna was selected which can be regarded as minimum compliant in terms of group delay variation and axial ratio for the L5 band. The performance for the L1 band is significantly better and not very conservative. The antenna was chosen as a model already exists for GPS L1 but not for any signals in the L5/E5a band in order to not get too optimistic results for the new frequency band.

Regarding the AGDV, it was decided to remove the AGDV from the observed errors. This was possible since the antennas flying on the test aircraft were measured in a compact test range and the aircraft attitude (and therefore the signal arrival angles) are known from the flight test installation. The AGDV was calibrated for mainly two reasons:

1. The nature of the error is more bias-like and slower changing than the multipath. Therefore the error characteristics are different and less well described by a standard deviation as a random error.

2. There are significant differences between different antennas in how much the group delay varies. Separating the budget for the antenna out of the multipath allows for leveraging the better performance of a better performing antenna (or also leveraging of potentially tighter future antenna specifications).

It should be noted, however, that the AGDV budget has to be considered and accounted for in any integrity concept if separated out of the multipath model.

\section{Receiver parameters:}

Background: For the receiver parameters, mainly the correlators and the receiver bandwidth have an influence on the tracking performance w.r.t. multipath errors. Airborne receivers generally use early-minus-late correlators, according to standards also double-delta correlators could be used.

Standards: Existing single-frequency standards leave a design space for the choice of bandwidth and correlator spacing. This is also foreseen in the future DFMC MOPS, however with a more restricted area of combinations. For the L5-band the correlator spacing is about 10-times the one for the L1-band [4]. The design space is depicted in Figure 2

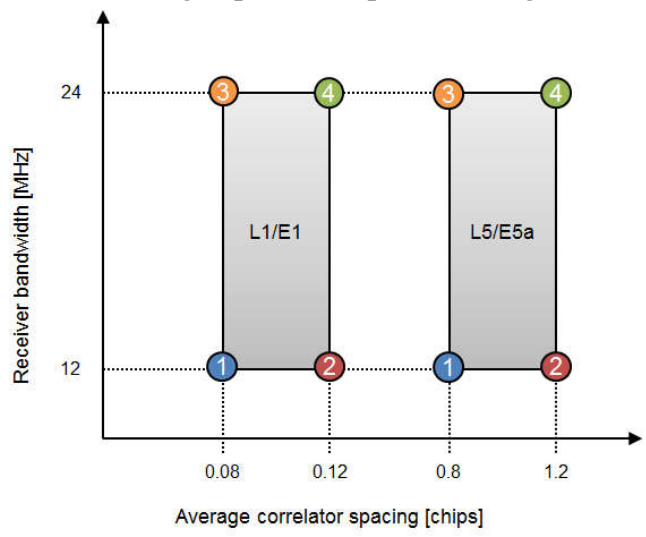

Figure 2 Receiver parameter design space 
Design choices in the project: For the project most of the data is recorded with an RF recorder and later replayed to receivers. This allows variation of the receiver parameters and evaluation of the impact on the resulting errors. Figure 3 shows the results of a replay to a commercial receiver where the correlator spacing and bandwidth can be configured. The curves are derived for the four corners of the design spaces for the different frequencies and constellations after 100s smoothing. Within the design space the differences are very small and can be reasonably well bounded with the same model.

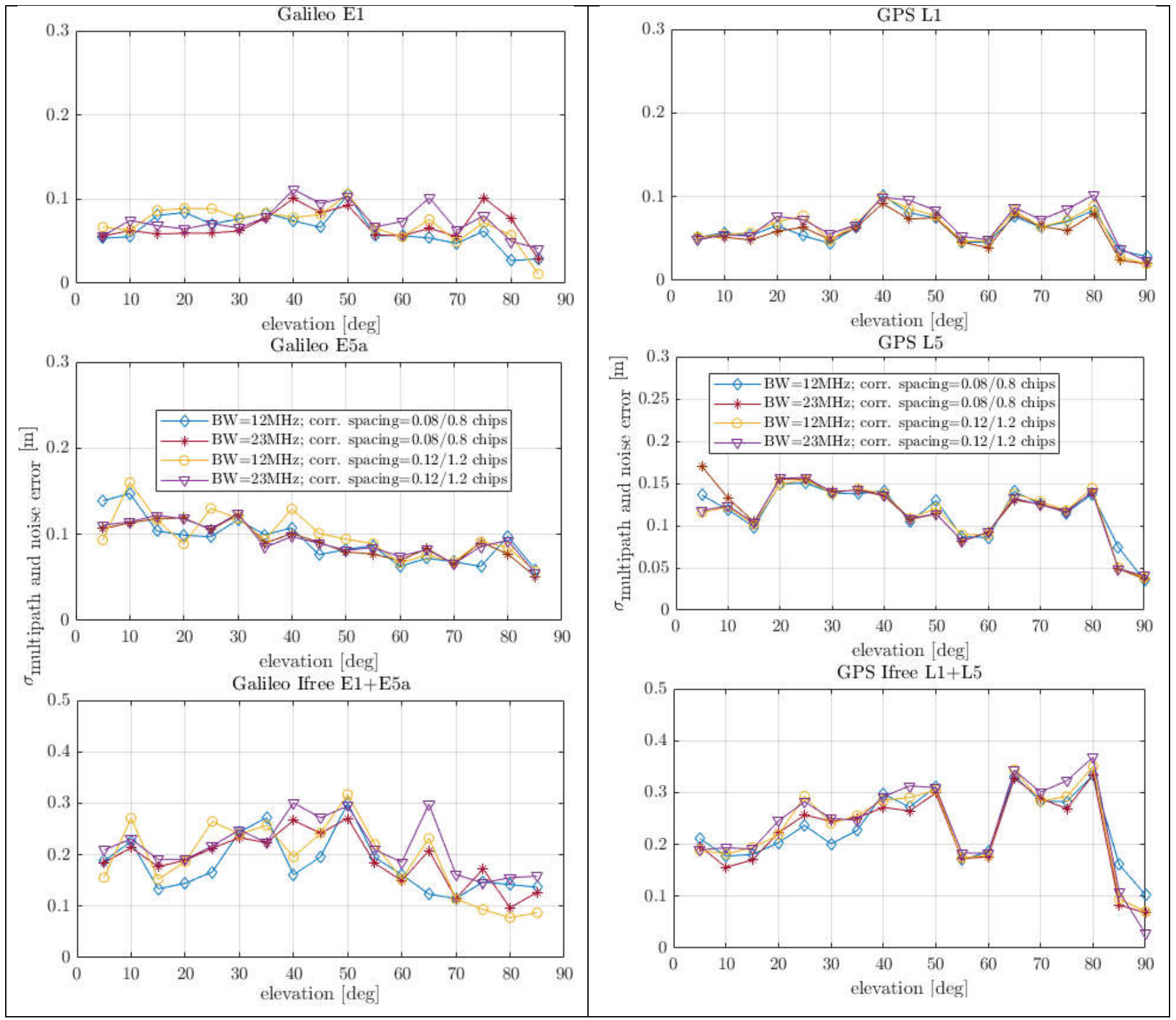

Figure 3 Impact of correlator spacing and receiver bandwidth on multipath performance. The left plots show the results for Galileo, the plots on the right the results for GPS

\section{Smoothing and smoothing time constant:}

Background: Code-carrier smoothing is an effective means to remove large parts of the noise and multipath. Furthermore, changes in the group delay errors are partially removed, depending on how fast the errors change. This depends on much the aircraft is maneuvering and how strongly the biases vary w.r.t. the signal arrival angle. For dual-frequency techniques that are under 
development at the moment, it is not decided whether the smoothing time constant will be increased to 600 seconds to further reduce residual errors.

Standards: Currently, all navigation techniques employ a Hatch-filter with a smoothing time constant of 100s. The only exception is the GBAS Approach Service Type D, which will be basing the position estimation on measurements with only 30 s of smoothing as part of the mitigation strategy against ionospheric gradients. However, for integrity purposes the 100s model is still used (another parameter accounting for the noise difference between 30s and 100s smoothed measurements is added).

ED-259 also specifies that "the multipath and antenna group delay variation error for unsmoothed measurements is described by [..] a standard deviation ten times greater than for 100-second smoothed measurements" [4]. For the transient phase between unsmoothed and steady-state no specifications are currently defined.

Furthermore, all navigation techniques assume that a Hatch filter is used. For future dual-frequency methods, some other filter techniques might be developed in order to be more robust against cycle slips or other signal losses (e.g. due to ionospheric effects).

Design choices in the project: For the time being, all results are based on 100s Hatch filtered data. The smoothing time constant is, however, just a parameter in the evaluation tools and can be easily changed if necessary.

\section{CONCLUSIONS AND STANDARDIZATION ACTIVITIES}

An important aspect of the project is the support to standardization of the new multipath models. Based on the flight data and supported by simulations, initial multipath models for Galileo E1, Galileo E5a and GPS L5 are suggested and will be further validated with more flight test data.

It was shown that the antenna is a major contributor to the overall error budget. For standardization purposes, a model that covers all MOPS compliant antennas should be developed. However, it was also observed that significant differences exist for different antennas on the market. The new airborne error models are therefore also proposed in such a way that it might be possible to exploit better performance in terms of AGDV in the future by separating the effect out and accounting for it in a better suited way. The models are all derived based on 100s Hatch filtered data. However, related work is ongoing and investigating the transient phase of the smoothing filter. This may facilitate a more appropriate error bounding for inclusion of satellites in the position solution before filter convergence. For different types of filters or different smoothing time constants new models may be easily developed based on the dataset of raw measurements collected in the project.

The definition of these new models is an important milestone in enabling the use of Galileo and dual-frequency techniques for aviation.

\section{ACKNOWLEDGMENTS}

The work presented in this paper was carried out in the frame of the project DUFMAN funded by the European Commission (DGGROW / JRC). Opinions expressed herein are those of the contractor only and do not represent the contracting authorities' official position. 


\section{REFERENCES}

[1] Minimum Operational Performance Standards for GPS Local Area Augmentation System Airborne Equipment, DO-253D, RTCA 2017

[2] Annex 10 to the Convention on International Civil Aviation, Aeronautical Telecommunications, Volume I Radio Navigation Aids, Seventh Edition, ICAO, July 2018

[3] Minimum Operational Performance Standards for Global Positioning System/Satellite Based Augmentation System Airborne Equipment, DO-229E, RTCA 2016

[4] Minimum Operational Performance Standards for Galileo / Global Positioning System / Satellite-Based Augmentation System Airborne Equipment, ED-259, Eurocae 2019

[5] Murphy T., Harris M., Booth J., Geren P., Pankaskie T., Clark B., Burns J., Urda T.- Results from the program for the investigation of airborne multipath errors, Proc. of ION NTM, 24-26 January 2005, San Diego, CA, USA

[6] Murphy T., Harris M., Geren P., Pankaskie T., Clark B., Burns J., - More results from the investigation of airborne multipath errors, Proc. of ION ITM, 13016 September 2005, Long Beach, CA, USA

[7] M.-S. Circiu, S. Caizzone, M. Felux, C. Enneking, M. Meurer, "Improved Airborne Multipath Modelling", Proceedings of ION GNSS+ 2018, Miami, FL, USA, September 2018

[8] M. Felux, M.-S. Circiu, S. Caizzone, C. Enneking, F. Fohlmeister, M. Rippl, "Towards Airborne Multipath Models for Dual Constellation and Dual-frequency GNSS", Proceedings of the 2019 International Technical Meeting of The Institute of Navigation, Reston, VI, UDA, January 2019

[9] Caizzone, S.; Circiu, M.-S.; Elmarissi, W.; Enneking, C.; Winterstein, A., „Airborne Antenna and Multipath Error Characterization for DFMC Error Standardization“, Proc. of ION GNSS+ 2019, Miami (USA), Sept. 2019

[10] Circiu, M.-S., Felux, M., Thoelert, S., Antreich, F., Vergara, M., Sgammini, M., Enneking, C., Pullen, S. (2015) Evaluation of GPS L5 and Galileo E1 and E5a Performance for Future Multi Frequency and Multi Constellation GBAS. Proc. of the ION International Technical Meeting ION GNSS, Dana Point, CA, US.

[11] Circiu, M.-S., Felux, M., Belabbas, B. Meurer, M., Lee, J., Kim, M., Pullen, S. (2015) Evaluation of GPS L5, Galileo E1 and Galileo E5a Performance in Flight Trials for Multi Frequency Multi Constellation GBAS. Proceedings of ION GNSS+ 2015. ION GNSS+ 2015, 14-18 September 2015, Tampa, Florida, US.

[12] Minimum Operational Performance Standards (MOPS) for Global Navigation Satellite System (GNSS) Airborne Active Antenna Equipment for the L1 Frequency Band, DO-301, RTCA 2006

[13] MOPS for GNSS Airborne Active Antenna Equipment for the L1/E1 and L5/E5a Frequency Bands, DO-373, RTCA 2018 